\title{
Parámetros Técnicos de Captura en Instrumentos Musicales Percutidos del Folclor Colombiano Para su Uso en Bancos Virtuales de Sonidos
}

\section{Technical Parameters Casings Capture of Colombian Folklore Musical InStRUMentS For Use In VirTual Sound BankS}

\author{
Carlos Andrés Caballero Parra ${ }^{1}$, Jamir Mauricio Moreno Espinal ${ }^{2}$ \\ Facultad de Artes y Humanidades, Instituto Tecnológico Metropolitano \\ Medellín, Colombia \\ ${ }^{1}$ carloscaballero@itm.edu.co \\ ${ }^{2}$ mauriciomoreno@itm.edu.co
}

\section{RESUMEN}

En este artículo se describirá la forma correcta y apropiada para la conceptualización técnica de la captura de los diferentes sonidos que caracterizan los instrumentos de percusión del folclor colombiano, teniendo en cuenta los parámetros propios de cada uno de estos y los formatos usados en los instrumentos y bancos virtuales de sonido en la actualidad. Las diferentes pruebas y análisis permiten demostrar que es necesario obtener una captura amplia, con micrófonos en su mayoría de respuesta plana, con formatos de conversión a alta resolución entre 24 bits y $96 \mathrm{kHz}$, tomas en estéreo tanto cercanas como lejanas y en ambientes acústicamente controlados y preparados para la correcta interpretación del instrumento.

Palabras clave: folclor, instrumentos virtuales, percusión.

\section{Abstract}

Through this article we describe the correct and appropriate for conceptualizing technique in capturing the different sounds that characterize Colombian folklore percussion instruments, taking into account parameters of the instruments themselves and the formats used in the instruments and virtual banks currently sound. The various tests and trials necessary to demonstrate that capture a wide, microphones mostly flat response, with conversion formats at high resolution between $24-b i t$ and $96 \mathrm{kHz}$, stereo jacks on both near and far and acoustically controlled environments prepared for the correct interpretation of the instrument.

Keywords: folklore, virtual instruments, percussion. 


\section{IntRODUCCIÓN}

La actual evolución de los procesos de captura y conversión de audio a un medio digital han permitido el rápido crecimiento y proliferación de sonidos sampleados o muestreados que simulan el instrumento interpretado en la realidad, aspecto muy diferente al concepto de síntesis de audio digital o analógica, el cual se encarga de simular por medio de diferentes procesos electrónicos y digitales un instrumento de la realidad tratando de representar su envolvente acústica con el uso de osciladores de frecuencia y generadores de tono, entre otros elementos.

Estos factores tecnológicos han permitido de muchas formas que la mayoría de culturas se interesen en la conservación y divulgación de sus instrumentos folclóricos debido a que la industria mundial da prioridad a los instrumentos que componen los ensambles clásicos y a los géneros musicales populares y tradicionales, como es el caso de la orquesta sinfónica o las agrupaciones de rock-pop con toda su gama de instrumentos; también es muy común encontrar instrumentos de música latina con raíces afro descendientes. Este artículo, entonces, pretende dar cuenta de la forma ideal de captura y entrega de los instrumentos tradicionales del folclor colombiano enmarcados cada uno de ellos en el ritmo propio de la región a la que pertenecen.

Los factores tenidos en cuenta darán como resultado un sonido que puede utilizarse de varias maneras y convertirse a varios formatos para su uso en diferentes plataformas de producción musical; para ello, se consideran detalles, tales como, la interpretación del músico en sesión y como solista, así como el espacio acústico en donde se interpreta, pues los sonidos pertenecen definitivamente al ambiente en el cual se desarrollan. Esto quiere decir que un instrumento se debe capturar en un espacio igual o similar al de su origen, siempre y cuando el resultado técnico de la muestra sea adaptable o manipulable y no sufra grandes variaciones sonoras que hagan difícil su entendimiento y escucha dentro de un contexto o interpretación musical. Con esto, podemos certificar que las muestras o samplers van a ser utilizados en cualquier manifestación o uso musical dentro de los parámetros técnicos necesarios de acuerdo con el estilo empleado; es decir, no tendría ningún sentido utilizar un fragmento musical en forma de loop, o bucle si este se deteriora al programarse en un tempo diferente o lejano al original [1].

Otras de las cuestiones son la ubicación y uso de los micrófonos de acuerdo con su respuesta direccional, el tipo de transducción y respuesta en frecuencia y el lugar o distancia con respecto a la fuente o instrumento en el cual se ubiquen, para que, de esta manera, puedan simular un espacio real y se puedan combinar con tomas cercanas que usualmente son frías y secas y que en su mayoría no respetan la respuesta real en frecuencia de cada instrumento, deformando así el balance tonal del mismo [2].

Para terminar, se debe tener en cuenta la calidad de la muestra tanto en el formato de captura como en el de entrega de tal forma que no sufran deterioro en su manipulación y a la vez sean maleables en su uso y se acomoden a diferentes plataformas. Esto es importante, pues en la actualidad existen un sin número de herramientas de trabajo con superficies de trabajo móviles aplicables a juegos y manipulación multimedia como también la posibilidad de utilizarse en grandes producciones musicales con sistemas de grabación mucho más complejos [2].

\section{Metodología}

La metodología para lograr los resultados esperados en este caso se divide en dos elementos básicos: una primera instancia, se basa en el 
análisis de las consideraciones técnicas de las tomas y capturas para seleccionar las mejores y más efectivas de acuerdo con el producto buscado y, una segunda etapa, que consiste en el análisis de interpretación musical de cada una de ellas. Por medio del uso de diferentes técnicas de grabación se puede establecer la mejor manera de realizar las tomas o muestras musicales teniendo en cuenta el objetivo final, el cual será el de tener un sonido muy parecido o aproximado al ejecutado por los músicos de sesión en condiciones interpretativas reales lo que es necesario debido a que la mayoría de instrumentos midi del medio no tienen un sonido real y, por lo general, suenan frío y sin expresión, contrario a lo que se busca en cualquier interpretación musical [3].

Otra de las consideraciones, es la necesidad de tener un sonido de alta calidad que pueda ser utilizado por cualquier productor, músico o compositor para sus trabajos profesionales sin necesidad de recurrir a fragmentos de grabaciones hechas en contextos de producción musical tradicional, los cuales difieren de las condiciones de grabación realizadas por empresas dedicadas a la comercialización de sampler o sonidos muestreados.

Las técnicas utilizadas para la captura, en su mayoría, se realizan con pares de micrófonos en estéreo, pues de esta manera se llega mejor a la sensación psicoacústica de los seres humanos de escucha con dos oídos, a excepción de las tomas cercanas, las cuales se aplicaban a partes de los instrumentos que se debían resaltar mucho más pero que al final se sumaban a la toma estéreo general del instrumento: por otra parte, era necesario diferenciar las tomas que se utilizarían para simular espacios o ambientes determinados y las tomas que se usarían únicamente para ser interpretadas por medio de un bucle simple como acompañamiento instrumental.

En un principio es necesario tener en cuenta que los instrumentos musicales percutidos tienen una técnica de captura diferente a los instrumentos armónico-melódicos: por tanto, es importante diferenciar las consideraciones técnicas y las musicales.

\section{A. Consideraciones técnicas}

En términos generales las grabaciones musicales se deberían realizar con tomas hechas en sesión o ensamble musical pues de esta manera se garantiza la esencia del sonido en un contexto real musical y no por separado. Para tal fin, es necesario contar con un espacio grande que tenga la posibilidad de separar los instrumentos para evitar filtraciones en las tomas y que al mismo tiempo el músico sienta la interpretación de su compañero de grupo en tiempo real; este tipo de grabación, aunque ideal para capturar la esencia interpretativa de una agrupación o ritmo para ser usado como loop o bucle, presentó varios problemas técnicos al intentar capturar un instrumento para ser usado de manera aislada, por lo que se hizo necesario realizar una grabación instrumento por instrumento. Se recomienda, por ende, realizar tomas por separado de cada uno de los instrumentos con varios micrófonos que den cuenta de un espacio acústico real en combinación con tomas cercanas que definan mucho más la interpretación de los mismos [2].

Es importante mencionar que las capturas realizadas en sesión serían utilizadas para un banco de sonidos especial que se utilizaría básicamente en forma de bucle o loop y que serviría mucho para realizar composiciones, arreglos o pre producciones musicales. Los tipos de micrófonos que se utilizan en cada toma es otro factor importante por tener en cuenta. La selección de estos debe corresponder a su configuración, propiedades sónicas y al tipo de fuente o instrumento por capturar, de tal forma que a un instrumento con bastante presión sonora interpretado con algún tipo de baqueta o palo, y que debido a esto genera una sonoridad mucho más alta que un instrumento de percusión menor, le corresponde capturarse con un micrófono que resista grandes cantidades de presión sonora; por otra parte, los instrumentos de percusión menor, en algunos casos, son susceptibles de ser capturados por micrófonos de alta sensibilidad y así entregarían mucha más información en el proceso de captura [1].

En la tabla I se puede observar una referencia para cada instrumento y el tipo de micrófono sugerido para cada uno de ellos. 
TABLA I

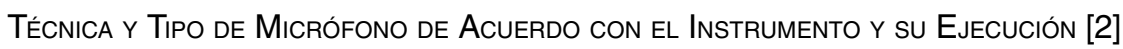

\begin{tabular}{|c|c|c|c|c|}
\hline \multicolumn{2}{|c|}{ Instrumento } & $\begin{array}{l}\text { Forma de } \\
\text { Ejecución }\end{array}$ & $\begin{array}{l}\text { Tipo de transducción/ } \\
\text { Tipo de diafragma }\end{array}$ & Tipo de toma \\
\hline $\begin{array}{l}\text { Región } \\
\text { Andina } \\
\text { Bambucc }\end{array}$ & $\begin{array}{l}\text { Bombo } \\
\text { andino }\end{array}$ & $\begin{array}{l}\text { Parche con } \\
\text { baqueta } \\
\text { Madera con } \\
\text { baqueta }\end{array}$ & $\begin{array}{l}\text { Micrófono dinámico/ } \\
\text { Grande } \\
\text { Micrófono condensador/ } \\
\text { Pequeño }\end{array}$ & $\begin{array}{l}\text { Cercana1 } \\
\text { Cercana con atenuador de } \\
\text { salida y filtro con corte de } \\
\text { bajos a } 100 \mathrm{~Hz}\end{array}$ \\
\hline & Carángano & Puño & $\begin{array}{l}\text { Micrófonos de } \\
\text { condensador }\end{array}$ & $\begin{array}{l}\text { Toma estéreo de acento } \\
\text { ORTF2 }\end{array}$ \\
\hline & Chucho & Manos & $\begin{array}{l}\text { Micrófonos de } \\
\text { condensador }\end{array}$ & Toma estéreo cercana en $X-Y$ \\
\hline & Esterilla & Manos & $\begin{array}{l}\text { Micrófonos de } \\
\text { condensador }\end{array}$ & Toma estéreo cercana en $X-Y$ \\
\hline & Marrana & Manos & $\begin{array}{l}\text { Micrófonos de } \\
\text { condensador }\end{array}$ & Toma estéreo cercana en $X-Y$ \\
\hline & Platillo & Baqueta & $\begin{array}{l}\text { Micrófono de } \\
\text { condensador/ pequeño }\end{array}$ & Cercana \\
\hline & \multirow[b]{2}{*}{ Redoblante } & \multirow[b]{2}{*}{$\begin{array}{l}\text { Baqueta en el par- } \\
\text { che superior }\end{array}$} & $\begin{array}{l}\text { Micrófono dinámico en el } \\
\text { parche superior }\end{array}$ & Cercana \\
\hline & & & $\begin{array}{l}\text { Micrófono de condensa- } \\
\text { dor/parche inferior }\end{array}$ & $\begin{array}{l}\text { Cercana con atenuador de } \\
\text { salida, filtro con corte de } \\
\text { bajos a } 100 \mathrm{~Hz} \text { e inversión } \\
\text { de fase }\end{array}$ \\
\hline \multirow{8}{*}{$\begin{array}{l}\text { Región } \\
\text { Andina } \\
\text { Pasillo }\end{array}$} & \multirow{2}{*}{$\begin{array}{l}\text { Bombo } \\
\text { andino }\end{array}$} & $\begin{array}{l}\text { Parche con } \\
\text { baqueta }\end{array}$ & $\begin{array}{l}\text { Micrófono dinámico/ } \\
\text { Grande }\end{array}$ & Cercana \\
\hline & & $\begin{array}{l}\text { Madera con } \\
\text { baqueta }\end{array}$ & $\begin{array}{l}\text { Micrófono condensador/ } \\
\text { Pequeño }\end{array}$ & $\begin{array}{l}\text { Cercana con atenuador de } \\
\text { salida y filtro con corte de } \\
\text { bajos a } 100 \mathrm{~Hz}\end{array}$ \\
\hline & Cucharas & Manos & $\begin{array}{l}\text { Micrófonos de } \\
\text { condensador }\end{array}$ & Toma estéreo cercana en $X-Y$ \\
\hline & Guacharaca & Manos & $\begin{array}{l}\text { Micrófonos de } \\
\text { condensador }\end{array}$ & Toma estéreo cercana en $X-Y$ \\
\hline & Mate & Manos & $\begin{array}{l}\text { Micrófonos de } \\
\text { condensador }\end{array}$ & Toma estéreo cercana en $X-Y$ \\
\hline & Platillos & Baqueta & $\begin{array}{l}\text { Micrófono de } \\
\text { condensador/ pequeño }\end{array}$ & Cercana \\
\hline & & & $\begin{array}{l}\text { Micrófono dinámico en el } \\
\text { parche superior }\end{array}$ & Cercana \\
\hline & Redoblante & $\begin{array}{l}\text { Baqueta en el } \\
\text { parche superior }\end{array}$ & $\begin{array}{l}\text { Micrófono de condensa- } \\
\text { dor/parche inferior }\end{array}$ & $\begin{array}{l}\text { Cercana con atenuador de } \\
\text { salida, filtro con corte de } \\
\text { bajos a } 100 \mathrm{~Hz} \text { e inversión } \\
\text { de fase }\end{array}$ \\
\hline
\end{tabular}




\begin{tabular}{|c|c|c|c|c|}
\hline \multicolumn{2}{|c|}{ Instrumento } & $\begin{array}{l}\text { Forma de } \\
\text { Ejecución }\end{array}$ & $\begin{array}{l}\text { Tipo de transducción/ } \\
\text { fipo de diafragma }\end{array}$ & Tipo de toma \\
\hline \multirow{6}{*}{$\begin{array}{l}\text { Región } \\
\text { Atlántica } \\
\text { Cumbia }\end{array}$} & Alegre & Mano & $\begin{array}{l}\text { Dinámico y condensador } \\
\text { en combinación de diafrag- } \\
\text { ma pequeño y grande }\end{array}$ & Cercana \\
\hline & Guache & Manos & $\begin{array}{l}\text { Micrófonos de } \\
\text { condensador }\end{array}$ & Toma estéreo cercana en X-Y \\
\hline & Llamador & Manos & $\begin{array}{l}\text { Dinámico y condensador } \\
\text { en combinación de diafrag- } \\
\text { ma pequeño y grande }\end{array}$ & Cercana \\
\hline & Maracón & Manos & $\begin{array}{l}\text { Micrófonos de } \\
\text { condensador }\end{array}$ & Toma estéreo cercana en X-Y \\
\hline & \multirow{2}{*}{ Tambor } & $\begin{array}{l}\text { Parche con } \\
\text { baqueta }\end{array}$ & $\begin{array}{l}\text { Micrófono dinámico/ } \\
\text { Grande }\end{array}$ & Cercana \\
\hline & & $\begin{array}{l}\text { Madera con } \\
\text { baqueta }\end{array}$ & $\begin{array}{l}\text { Micrófono condensador/ } \\
\text { Pequeño }\end{array}$ & $\begin{array}{l}\text { Cercana con atenuador de } \\
\text { salida y filtro con corte de } \\
\text { bajos a } 100 \mathrm{~Hz}\end{array}$ \\
\hline \multirow{5}{*}{$\begin{array}{l}\text { Región } \\
\text { Atlantica } \\
\text { Porro } \\
\text { Pelayero }\end{array}$} & \multirow[b]{2}{*}{ Bombo } & Parche con mano & $\begin{array}{l}\text { Micrófono dinámico/ } \\
\text { Grande }\end{array}$ & Cercana \\
\hline & & $\begin{array}{l}\text { Madera con ba- } \\
\text { queta }\end{array}$ & $\begin{array}{l}\text { Micrófono condensador/ } \\
\text { Pequeño }\end{array}$ & $\begin{array}{l}\text { Cercana con atenuador de } \\
\text { salida y filtro con corte de } \\
\text { bajos a } 100 \mathrm{~Hz}\end{array}$ \\
\hline & Platillo & Baqueta & $\begin{array}{l}\text { Micrófono de } \\
\text { condensador/ pequeño }\end{array}$ & Cercana \\
\hline & \multirow[b]{2}{*}{ Redoblante } & \multirow[b]{2}{*}{$\begin{array}{l}\text { Baqueta en el par- } \\
\text { che superior }\end{array}$} & $\begin{array}{l}\text { Micrófono dinámico en el } \\
\text { parche superior }\end{array}$ & Cercana \\
\hline & & & $\begin{array}{l}\text { Micrófono de condensa- } \\
\text { dor/parche inferior }\end{array}$ & $\begin{array}{l}\text { Cercana con atenuador de } \\
\text { salida, filtro con corte de } \\
\text { bajos a } 100 \mathrm{~Hz} \text { e inversión } \\
\text { de fase }\end{array}$ \\
\hline \multirow{2}{*}{$\begin{array}{l}\text { Región } \\
\text { Atlantica } \\
\text { Vallenato }\end{array}$} & Caja & Mano & $\begin{array}{l}\text { Dinámico y condensador } \\
\text { en combinación de diafrag- } \\
\text { ma pequeño y grande }\end{array}$ & Cercana \\
\hline & Guacharaca & Manos & $\begin{array}{l}\text { Micrófonos de } \\
\text { condensador }\end{array}$ & Toma estéreo cercana en $X-Y$ \\
\hline
\end{tabular}




\begin{tabular}{|c|c|c|c|c|}
\hline \multicolumn{2}{|c|}{ Instrumento } & $\begin{array}{l}\text { Forma de } \\
\text { Ejecución }\end{array}$ & $\begin{array}{l}\text { Tipo de transducción/ } \\
\text { Tipo de diafragma }\end{array}$ & Tipo de toma \\
\hline \multirow{8}{*}{$\begin{array}{l}\text { Región } \\
\text { Pacífica } \\
\text { Currulao }\end{array}$} & \multirow[b]{2}{*}{ Arrullador } & $\begin{array}{l}\text { Parche con ba- } \\
\text { queta }\end{array}$ & $\begin{array}{l}\text { Micrófono dinámico/ } \\
\text { Grande }\end{array}$ & Cercana \\
\hline & & $\begin{array}{l}\text { Madera con ba- } \\
\text { queta }\end{array}$ & $\begin{array}{l}\text { Micrófono condensador/ } \\
\text { Pequeño }\end{array}$ & $\begin{array}{l}\text { Cercana con atenuador de } \\
\text { salida y filtro con corte de } \\
\text { bajos a } 100 \mathrm{~Hz}\end{array}$ \\
\hline & \multirow[b]{2}{*}{ Bombo } & $\begin{array}{l}\text { Parche con ba- } \\
\text { queta }\end{array}$ & $\begin{array}{l}\text { Micrófono dinámico/ } \\
\text { Grande }\end{array}$ & Cercana \\
\hline & & $\begin{array}{l}\text { Madera con ba- } \\
\text { queta }\end{array}$ & $\begin{array}{l}\text { Micrófono condensador/ } \\
\text { Pequeño }\end{array}$ & $\begin{array}{l}\text { Cercana con atenuador de } \\
\text { salida y filtro con corte de } \\
\text { bajos a } 100 \mathrm{~Hz}\end{array}$ \\
\hline & $\begin{array}{l}\text { Cununo } \\
\text { Hembra }\end{array}$ & Manos & $\begin{array}{l}\text { Dinámico y condensador } \\
\text { en combinación de diafrag- } \\
\text { ma pequeño y grande }\end{array}$ & Cercana \\
\hline & $\begin{array}{l}\text { Cununo } \\
\text { Macho }\end{array}$ & Manos & $\begin{array}{l}\text { Dinámico y condensador } \\
\text { en combinación de diafrag- } \\
\text { ma pequeño y grande }\end{array}$ & Cercana \\
\hline & Guasá & Manos & $\begin{array}{l}\text { Micrófonos de } \\
\text { condensador }\end{array}$ & Toma estéreo cercana en $X-Y$ \\
\hline & Guasá 2 & Manos & $\begin{array}{l}\text { Micrófonos de } \\
\text { condensador }\end{array}$ & Toma estéreo cercana en X-Y \\
\hline \multirow{5}{*}{$\begin{array}{l}\text { Región } \\
\text { Pacífica } \\
\text { Porro } \\
\text { chocoano }\end{array}$} & \multirow[b]{2}{*}{ Bombo } & $\begin{array}{l}\text { Parche con } \\
\text { baqueta }\end{array}$ & $\begin{array}{l}\text { Micrófono dinámico/ } \\
\text { Grande }\end{array}$ & Cercana \\
\hline & & $\begin{array}{l}\text { Madera con } \\
\text { baqueta }\end{array}$ & $\begin{array}{l}\text { Micrófono condensador/ } \\
\text { Pequeño }\end{array}$ & $\begin{array}{l}\text { Cercana con atenuador de } \\
\text { salida y filtro con corte de } \\
\text { bajos a } 100 \mathrm{~Hz}\end{array}$ \\
\hline & \multirow[t]{2}{*}{ Pate Gallina } & \multirow[t]{2}{*}{ Baquetas } & $\begin{array}{l}\text { Micrófono de condensador } \\
\text { de diafragma pequeño para } \\
\text { el juego de campanas y } \\
\text { tas-tas }\end{array}$ & $\begin{array}{l}\text { Cercana con corte de bajos } \\
\text { a } 100 \mathrm{~Hz}\end{array}$ \\
\hline & & & $\begin{array}{l}\text { Micrófono dinámico dedi- } \\
\text { cado al redoblante }\end{array}$ & Cercana \\
\hline & $\begin{array}{l}\text { Platillos de } \\
\text { entrechoque }\end{array}$ & Manos & $\begin{array}{l}\text { Micrófonos de } \\
\text { condensador }\end{array}$ & Toma estéreo cercana en $X-Y$ \\
\hline $\begin{array}{l}\text { Región de } \\
\text { los Llanos } \\
\text { Orientales } \\
\text { Joropo }\end{array}$ & $\begin{array}{l}\text { Maracas } 0 \\
\text { capachos }\end{array}$ & Manos & $\begin{array}{l}\text { Micrófonos de condensa- } \\
\text { dor }\end{array}$ & Toma estéreo cercana en X-Y \\
\hline \multirow{2}{*}{$\begin{array}{l}\text { Región } \\
\text { insular } \\
\text { Calipso }\end{array}$} & Maracas & Manos & $\begin{array}{l}\text { Micrófonos de condensa- } \\
\text { dor }\end{array}$ & Toma estéreo cercana en X-Y \\
\hline & Quijada & Manos & $\begin{array}{l}\text { Micrófonos de condensa- } \\
\text { dor }\end{array}$ & Toma estéreo cercana en $X-Y$ \\
\hline
\end{tabular}

Fuente: los autores 
Como se puede evidenciar en la tabla I, las recomendaciones de los micrófonos utilizados se basan en sus propiedades y parámetros de tipo técnico; se abstiene de realizar una recomendación directa de referencias y marcas de micrófonos debido a que en gran parte lo que se pretende es trabajar con los transductores que se tengan a disposición, y mejor se enfoca en los parámetros necesarios de los procesos de captura. En todo caso es importante mencionar cuáles micrófonos fueron utilizados en este proceso. A continuación se presenta una lista con los micrófonos utilizados en la captura de los instrumentos percutidos del folclor colombiano escogidos para este proyecto [4], [5].

- Neumman U87

- AKG C-414

- Sennheiser MD-421

- Shure SM57

- AKG C-451B

- AKG D-112

- Shure Beta- 51A

Por otra parte, es importante que los instrumentos en ensamble musical se capturen teniendo en cuenta el lugar en el que se realiza su interpretación; es por esto, que se recomienda, entonces, realizar dichas tomas en lugares de buena acústica interna, de tal forma, que las tomas se puedan combinar entre las lejanas y las cercanas y así brindar una dimensión espacial real a las muestras de cada uno. En este caso, un estudio con cabina de grabación amplia, auditorio o teatro es el lugar ideal para la captura; teniendo en cuenta el aprovechamiento del espacio, se puede medir incluso el tiempo de reverberación, con el fin de que en el momento de la mezcla de cada una de las tomas, se pueda combinar la toma cercana del instrumento con la lejana, lo que tendría como resultado una muestra con sonido ambiente natural y no artificial. Lo anterior, teniendo en cuenta que en un futuro se pretende aportar cada toma a un software o motor (que funcione de manera autónoma), o esclavo de interpretación musical, también llamado sintetizador o instrumento virtual, el cual se encargaría de administrar cada una de las tomas realizadas, como suelen utilizarse ya por muchos sistemas de trabajo de audio digital [5].

\section{B. Consideraciones musicales}

Resulta importante realizar tomas con diferentes dinámicas y formas de interpretación. Cuando un instrumento tan simple como una guacharaca se interpreta con el trinchete o gancho hacia arriba genera un sonido diferente que cuando se interpreta hacia abajo; lo mismo sucede con el golpe de un tambor cununo, ya sea este con quemado o con la palma de la mano abierta. Con base en lo anterior, se hace necesario incluir cada una de estas interpretaciones y técnicas en tomas diferentes para ampliar la librería de los banco de sonidos. A continuación se presenta una lista de instrumentos con cada toma necesaria para su correcta interpretación musical de acuerdo con cada región [5]. 
TABLA ॥

Interpretación Musical Capturada Por Cada Instrumento

\begin{tabular}{|c|c|c|}
\hline Región & Instrumento & Interpretación capturada \\
\hline \multirow{12}{*}{ Andina- bambuco y pasillo } & Bombo & $\begin{array}{l}\text { Palo de bombo } \\
\text { Parche y madera } \\
\text { Parche }\end{array}$ \\
\hline & Carángano & $\begin{array}{l}\text { Puño } \\
\text { Vejiga } \\
\text { Boliillo }\end{array}$ \\
\hline & Chucho & $\begin{array}{l}\text { Mano derecha } \\
\text { Mano izquierda }\end{array}$ \\
\hline & Cucharas de palo & $\begin{array}{l}\text { Cuchara } 1 \text { grave } \\
2 \text { Aguda }\end{array}$ \\
\hline & Esterilla & $\begin{array}{l}\text { Apagado } \\
\text { Rasgado }\end{array}$ \\
\hline & Guacharaca & $\begin{array}{l}\text { Seco grave } \\
\text { Seco agudo } \\
\text { Rasgado }\end{array}$ \\
\hline & Gusano & $\begin{array}{l}\text { Largo } \\
\text { Corto }\end{array}$ \\
\hline & Mates & $\begin{array}{l}\text { Con rebote } \\
\text { Seco }\end{array}$ \\
\hline & Platillos de entrechoque & $\begin{array}{l}\text { Abierto } \\
\text { Cerrado }\end{array}$ \\
\hline & Puerca & $\begin{array}{l}\text { Sencillo } \\
\text { Largo }\end{array}$ \\
\hline & Quijada & $\begin{array}{l}\text { Diente ascendente } \\
\text { Diente descendente } \\
\text { Palo } \\
\text { Puño }\end{array}$ \\
\hline & Quiribillo & $\begin{array}{l}\text { Corto } \\
\text { Prolongado }\end{array}$ \\
\hline
\end{tabular}




\begin{tabular}{|c|c|c|}
\hline Región & Instrumento & Interpretación capturada \\
\hline \multirow{6}{*}{ Atlántica - Cumbia } & Alegre & $\begin{array}{l}\text { Abierto centro } \\
\text { Abierto sencillo } \\
\text { Centro abierto } \\
\text { Centro cerrado } \\
\text { Golpe seco } \\
\text { Puño } \\
\text { Quemado abierto } \\
\text { Quemado sencillo }\end{array}$ \\
\hline & Guache & $\begin{array}{l}\text { Redoble compuesto } \\
\text { Redoble sencillo } \\
\text { Seco }\end{array}$ \\
\hline & Llamador & $\begin{array}{l}\text { Abierto sencillo } \\
\text { Abierto con baqueta } \\
\text { Aro } \\
\text { Quemado con baqueta } \\
\text { Quemado sencillo }\end{array}$ \\
\hline & Maracón & $\begin{array}{l}\text { Sencillo } \\
\text { Redoble 1, 2, } 3 \text { y } 4\end{array}$ \\
\hline & Tabla & Sencillo \\
\hline & Tambor & $\begin{array}{l}\text { Apagado } \\
\text { Madera } \\
\text { Parche } \\
\text { Parche y madera }\end{array}$ \\
\hline \multirow{3}{*}{ Atlántica - Porro Pelayero } & Bombo & $\begin{array}{l}\text { Apagado } \\
\text { Parche } \\
\text { Parche y apagado }\end{array}$ \\
\hline & Platillos & $\begin{array}{l}\text { Abierto } \\
\text { Cerrado } \\
\text { Medio abierto }\end{array}$ \\
\hline & Redoblante & $\begin{array}{l}\text { Sencillo } \\
\text { Redoble corto } \\
\text { Redoble largo espaciado } \\
\text { Redoble largo denso } \\
\text { Redoble seco } \\
\text { Ring shot }\end{array}$ \\
\hline \multirow[t]{2}{*}{ Atlántica - Vallenato } & Caja & $\begin{array}{l}\text { Abierto } \\
\text { Apagado } \\
\text { Quemado } \\
\text { Redoble }\end{array}$ \\
\hline & Guacharaca & $\begin{array}{l}\text { Abajo débil } \\
\text { Abajo } \\
\text { Arriba débil }\end{array}$ \\
\hline
\end{tabular}




\begin{tabular}{|c|c|c|}
\hline Región & Instrumento & Interpretación capturada \\
\hline \multirow{3}{*}{ Pacífica - porro chocoano } & Bombo & $\begin{array}{l}\text { Apagado y madera } \\
\text { Apagado } \\
\text { Madera } \\
\text { Parche y madera } \\
\text { Parche }\end{array}$ \\
\hline & Pate-Gallina & $\begin{array}{l}\text { Campana } \\
\text { Cencerro } \\
\text { Tas-Tas } \\
\text { Redoblante } \\
\text { Redoble sencillo } \\
\text { Rebote }\end{array}$ \\
\hline & Platillos & $\begin{array}{l}\text { Abierto } \\
\text { Cerrado } \\
\text { Medio abierto }\end{array}$ \\
\hline \multirow{5}{*}{ Pacífica Sur - currulao } & Bombo Arrullador & $\begin{array}{l}\text { Apagado } \\
\text { Madera } \\
\text { Parche y madera } \\
\text { Parche }\end{array}$ \\
\hline & Bombo Golpeador & $\begin{array}{l}\text { Apagado } \\
\text { Madera } \\
\text { Parche y madera } \\
\text { Parche }\end{array}$ \\
\hline & Cununo Hembra & \begin{tabular}{|l} 
Abierto \\
Quemado
\end{tabular} \\
\hline & Cununo Macho & \begin{tabular}{|l} 
Abierto \\
Quemado
\end{tabular} \\
\hline & Guasá & $\begin{array}{l}\text { Sencillo agudo } \\
\text { Sencillo grave } \\
\text { Redoble } \\
\text { Regado }\end{array}$ \\
\hline Llanos Orientales & Capachos & $\begin{array}{l}\text { Seco } 1 \text { y } 2 \\
\text { Redoble }\end{array}$ \\
\hline \multirow{2}{*}{ Insular } & Maracas & $\begin{array}{l}\text { Sencillo } \\
\text { Redoble } 1 \text { y } 2\end{array}$ \\
\hline & Quijada & $\begin{array}{l}\text { Abajo } \\
\text { Débiles } \\
\text { Puño }\end{array}$ \\
\hline
\end{tabular}

En cuanto al carácter musical de cada interpretación, en la música colombiana existe una variedad de ritmos en diferentes compases y velocidades, así que la importancia de tener un concepto claro de cómo se debe interpretar musicalmente cada instrumento es fundamental para el correcto desarrollo de cada muestra. Por ejemplo, un ritmo como la cumbia de la Región Atlántica tiene el golpe del tambor o bombo en el cuarto tiempo del compás; este sonido por su naturaleza y dinámica interna se prolonga en el tiempo mucho más allá del inicio del compás ya que tiene una relajación y un decaimiento largo. Así, cuando se piensa en tener un ritmo desde el primer tiempo del compás, es necesario realizar dos tomas: una que sea desde el primer tiempo del compás y que se pueda utilizar como muestra de inicio de compás en cada forma o ritmo musical y otra que se utilice para la marcha 
continua del instrumento a través de la canción, muy parecido a lo que ocurre con los samplers y loops de los ritmos con clave afro-cubana, los cuales se interpretan en clave de 3 por 2 y luego se agrega otro compás iniciando con la clave en 2 , con el fin de que el mismo sampler pueda ser usado en cualquiera de los dos casos [5].

Vale la pena mencionar, adicionalmente, la gran cantidad de diferentes interpretaciones que se pueden capturar en los instrumentos más representativos de las regiones; cada instrumento tiene sus características interpretativas manifestadas en partes del mismo instrumento, en la forma del golpe con la mano o con baqueta. Esto determina la cantidad de muestras por instrumento. Luego de determinada cada muestra es necesario establecer la dinámica interpretativa de cada una de ellas, con el objetivo de definir los niveles de fuerza en la ejecución cuando se va a utilizar el registro me- diante un controlador midi, o los llamados niveles de velocidad o velocity, presentes en todos los secuenciadores midi, programas musicales, sintetizadores y demás instrumentos con esta característica. Por último, se muestra el carácter rítmico el cual va relacionado directamente con la interpretación con anacrusa o tética. Esto es importante para obtener una muestra adicional que servirá a futuro al compositor o productor musical; vale la pena mencionar también que cada tabla posee el tempo de interpretación musical característica de cada ritmo y la unidad de compás.

En la tabla III se presentan los parámetros que deben contener las tablas relacionadas con la especificidad interpretativa de cada instrumento en particular regido por parámetros estrictamente musicales y estilísticos de acuerdo con el ritmo o aire tradicional registrado y su región geográfica a la que pertenecen.

TABLA III

Consideraciones Interpretativas Región Andina Colombiana

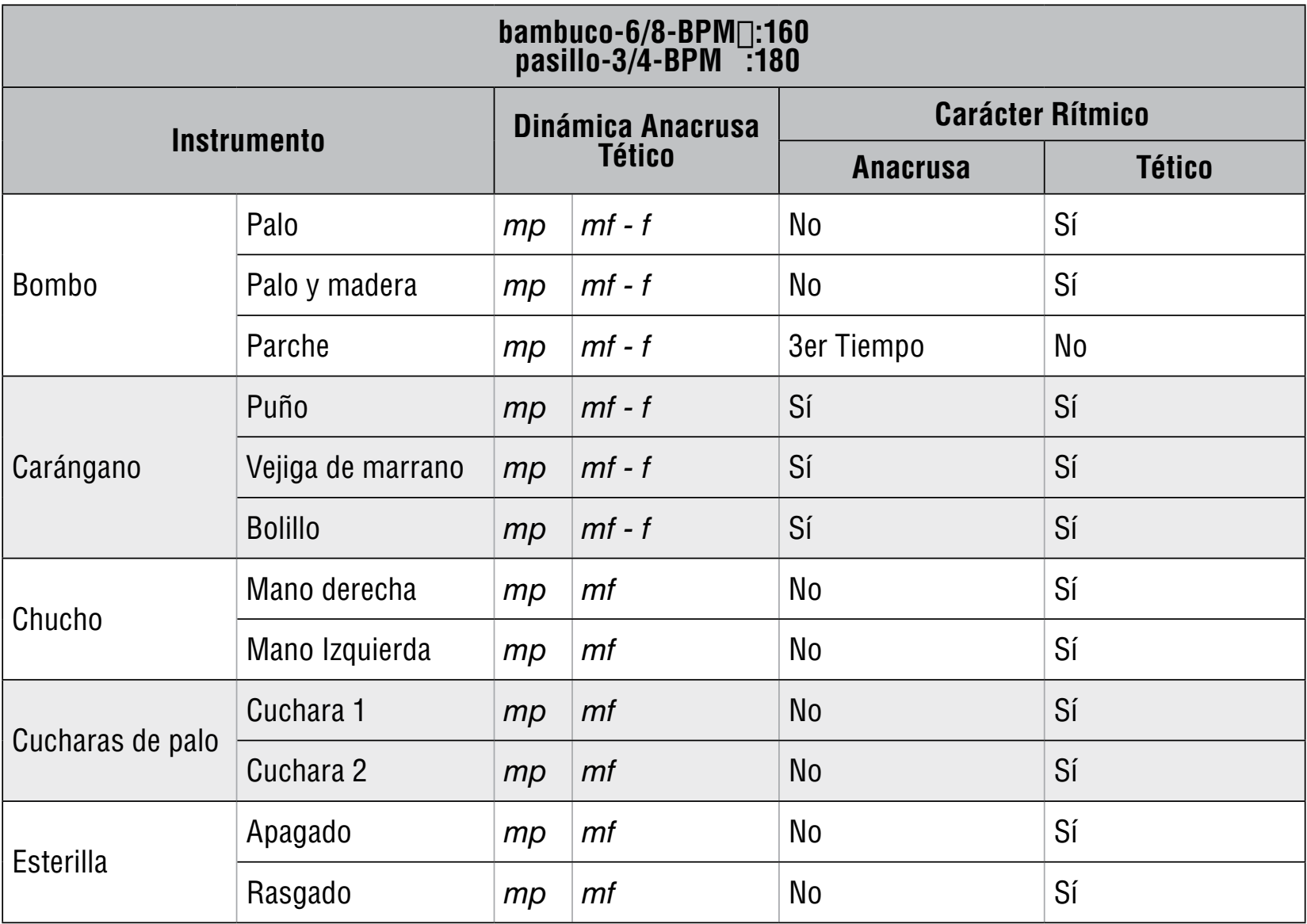




\begin{tabular}{|c|c|c|c|c|c|}
\hline \multicolumn{6}{|c|}{$\begin{array}{c}\text { bambuco-6/8-BPM } \square: 160 \\
\text { pasillo-3/4-BPM } \bowtie: 180\end{array}$} \\
\hline \multirow{3}{*}{ Guacharaca } & Seco 1 & $m p$ & $m f-f$ & Sí & Sí \\
\hline & Seco 2 & $m p$ & $m f-f$ & Sí & Sí \\
\hline & Rasgado & $m p$ & $m f-f$ & $\begin{array}{l}\text { 3er } \\
\text { Tiempo }\end{array}$ & Sí \\
\hline \multirow{2}{*}{ Gusano } & 1 & $m p$ & $m f$ & No & Sí \\
\hline & 2 & $m p$ & $m f$ & No & Sí \\
\hline \multirow{2}{*}{ Mates } & 1 & $m p$ & $m f$ & No & Sí \\
\hline & 2 & $m p$ & $m f$ & 3er Tiempo & No \\
\hline \multirow{2}{*}{ Platillos } & Abierto & $m p$ & $m f-f$ & $\begin{array}{l}\text { 3er } \\
\text { Tiempo }\end{array}$ & No \\
\hline & Cerrado & $m p$ & $m f-f$ & No & Sí \\
\hline \multirow{4}{*}{ Quijada } & Diente ascendente & $m p$ & $m f-f$ & Sí & Sí \\
\hline & Diente descendente & $m p$ & $m f-f$ & Sí & Sí \\
\hline & Palo & $m p$ & $m f-f$ & Sí & Sí \\
\hline & Puño & $m p$ & $m f-f$ & 3er Tiempo & Sí \\
\hline \multirow{2}{*}{ Quiribillo } & 1 & $m p$ & $m f$ & No & Sí \\
\hline & 2 & $m p$ & $m f$ & No & Sí \\
\hline \multirow{2}{*}{ Redoblante } & Sencillo & $m p$ & $m f-f$ & Sí & Sí \\
\hline & Redoble & $m p$ & $m f-f$ & 3er Tiempo & No \\
\hline
\end{tabular}

Igualmente, se deben tener las especificaciones técnicas para el resto de los aires tradicionales del folclor colombiano.

\section{Resultados}

El resultado obtenido de acuerdo con la metodología propuesta en la parte técnica arrojó muestras que dan cuenta de una captura de calidad y de una gran cantidad de información, la cual se seleccionó de tal manera que pudiera sintetizarse en bancos definidos y catalogados y que, a su vez, pudiesen ser utilizados como insumo para cualquier producción musical dependiendo del formato de destino; es así como se pudo establecer al final del proceso tres productos tangibles: el primero, fue una base de datos que contiene cuatro diferentes formatos de destino con información específica de acuerdo con la plataforma de trabajo; el segundo, fue un fonograma el cual contiene en formato tradicional de audio todas y cada una de las tomas registradas y el tercero, es una aplicación móvil que hace parte del proceso de divulgación y apropiación social del proyecto. En seguida se presenta cada uno de ellos.

\section{A. Base de datos-soporte lógico [6]}

De cada uno de los aires registrados se sacaron cuatro diferentes formatos de destino, los cuales fueron organizados y catalogados para que se pudieran usar de forma sencilla y, además, sirvieran de insumo para diferentes plataformas. Cabe 
recordar los aires que fueron seleccionados para este proyecto.

- Bambuco

- Calipso

- Cumbia

- Currulao

- Joropo

- Pasillo

- Pelayera

- Porro chocoano

- Vallenato

De cada uno de ellos se obtuvo información en los siguientes formatos [8]:

Wave (.wav): este es probablemente el formato más útil de trabajo debido a que no se encuentra comprimido como sí lo pueden estar los demás formatos de audio; la resolución final presentada es de $44.1 \mathrm{kHz}$ y $16 \mathrm{bits}$. Sin embargo, cabe recordar que las capturas fueron realizadas a $96 \mathrm{kHz}$ y 24bits en sus sesiones originales, con el ánimo de tener insumos que se deriven en otras aplicaciones y presentaciones.

Sound Fonts (.SF2): este formato resulta bastante útil a la hora de trabajar en formatos midi con algunas plataformas como Sonar y
Reason; sin embargo, es importante recalcar que el archivo sufre una compresión en el momento de ser convertido a dicho formato mediante la aplicación "Recycle" utilizada para este propósito.

Midi (.mid): este formato se hace necesario a la hora de hacer programaciones desde cualquier secuenciador o programa musical, ya que es el encargado de controlar precisamente cualquier muestra o sampler. Además, posee las características y propiedades interpretativas propias del sistema midi, tales como velocity, aftertouch, sensitivity, etc.

RecycleRex (.rx2): este formato propio de la plataforma de la compañía Propellerhead, llamada Reason, tiene la posibilidad de registrar, no solamente el sonido básico, sino también el bucle o loop con la ventaja de tener propiedades de audio elástico, lo que facilita el trabajo en interpretaciones musicales con diferentes tiempos. No obstante, es importante recordar que la modificación muy amplia del tiempo de los registros originales puede ocasionar distorsión y deformación de la señal original.

En la Tabla IV se muestran los resultados de la base de datos obtenida.

TABLA IV

Base de Datos Obtenida [6]

\begin{tabular}{|l|l|l|l|l|}
\hline $\begin{array}{c}\text { Banco de } \\
\text { sonidos }\end{array}$ & \multicolumn{1}{|c|}{ RecycleRex file } & \multicolumn{1}{|c|}{ Midi file } & \multicolumn{1}{|c|}{ Soundfont } & \multicolumn{1}{|c|}{ Wave (microsoft) } \\
& Bombo_bpm160.rx2 & Bombo_bpm160.mid & $\begin{array}{l}\text { Bombo_bpm160. } \\
\text { sf2 }\end{array}$ & Bombo_bpm160.wav \\
\cline { 2 - 5 } & $\begin{array}{l}\text { Carángano_bpm160. } \\
\text { rx2 }\end{array}$ & Carángano_bpm160.mid & $\begin{array}{l}\text { Carángano } \\
\text { bpm160.sf2 }\end{array}$ & $\begin{array}{l}\text { Carángano_bpm160. } \\
\text { wav }\end{array}$ \\
\cline { 2 - 6 } & Chucho_bpm160.rx2 & Chucho_bpm160.mid & $\begin{array}{l}\text { Chucho_bpm160. } \\
\text { sf2 }\end{array}$ & Chucho_bpm160.wav \\
\cline { 2 - 6 } Bambuco & Esterilla_bpm.rx2 & Esterilla_bpm.mid & Esterilla_bpm.sf2 & Esterilla_bpm.wav \\
\cline { 2 - 6 } & Marrrana_bpm.rx2 & Marrrana_bpm.mid & $\begin{array}{l}\text { Marrana_bpm. } \\
\text { sf2 }\end{array}$ & Marrrana_bpm.wav \\
\cline { 2 - 6 } & Platillo_bpm160.rx2 & Platillo_bpm160.mid & $\begin{array}{l}\text { Platillo_bpm160. } \\
\text { sf2 }\end{array}$ & Platillo_bpm160.wav \\
\cline { 2 - 6 } & $\begin{array}{l}\text { Redoblante_ } \\
\text { bpm160.rx2 }\end{array}$ & $\begin{array}{l}\text { Redoblante_bpm160. } \\
\text { mid }\end{array}$ & $\begin{array}{l}\text { Redoblante_ } \\
\text { bpm160.sf2 }\end{array}$ & $\begin{array}{l}\text { Redoblante_bpm160. } \\
\text { wav }\end{array}$ \\
\hline
\end{tabular}




\begin{tabular}{|c|c|c|c|c|}
\hline $\begin{array}{c}\text { Banco de } \\
\text { sonidos }\end{array}$ & RecycleRex file & Midi file & Soundfont & Wave (microsoft) \\
\hline \multirow{2}{*}{ Calipso } & $\begin{array}{l}\text { Maracas_bpm210. } \\
\text { rx2 }\end{array}$ & Maracas_bpm210.mid & $\begin{array}{l}\text { Maracas } \\
\text { bpm210.sf2 }\end{array}$ & Maracas_bpm210.wav \\
\hline & Quijada_bpm210.rx2 & Quijada_bpm210.mid & $\begin{array}{l}\text { Quijada_bpm210. } \\
\text { sf2 }\end{array}$ & Quijada_bpm210.wav \\
\hline \multirow{4}{*}{ Cumbia } & Guache_bpm185.rx2 & Guache_bpm185.mid & $\begin{array}{l}\text { Guache_bpm185. } \\
\text { sf2 }\end{array}$ & Guache_bpm185.wav \\
\hline & $\begin{array}{l}\text { Llamador_bpm185. } \\
\text { rx2 }\end{array}$ & Llamador_bpm185.mid & $\begin{array}{l}\text { Llamador } \\
\text { bpm185.sf2 }\end{array}$ & Llamador_bpm185.wav \\
\hline & $\begin{array}{l}\text { Maracón_bpm185. } \\
\text { rx2 }\end{array}$ & Maracón_bpm185.mid & $\begin{array}{l}\text { Maracón } \\
\text { bpm185.sf2 }\end{array}$ & Maracón_bpm185.wav \\
\hline & Tambor_bpm185.rx2 & Tambor_bpm185.mid & $\begin{array}{l}\text { Tambor_bpm185. } \\
\text { sf2 }\end{array}$ & Tambor_bpm185.wav \\
\hline \multirow{6}{*}{ Currulao } & $\begin{array}{l}\text { Arrullador_bpm140. } \\
\text { rx2 }\end{array}$ & Arrullador_bpm140.mid & $\begin{array}{l}\text { Arrullador } \\
\text { bpm140.sf2 }\end{array}$ & Arrullador_bpm140.wav \\
\hline & Bombo_bpm140.rx2 & Bombo_bpm140.mid & $\begin{array}{l}\text { Bombo_bpm140. } \\
\text { sf2 }\end{array}$ & Bombo_bpm140.wav \\
\hline & $\begin{array}{l}\text { Cununo hembra } \\
\text { bpm140.rx2 }\end{array}$ & $\begin{array}{l}\text { Cununo hembra } \\
\text { bpm140.mid }\end{array}$ & $\begin{array}{l}\text { Cununo hembra } \\
\text { bpm140.sf2 }\end{array}$ & $\begin{array}{l}\text { Cununo hembra } \\
\text { bpm140.wav }\end{array}$ \\
\hline & $\begin{array}{l}\text { Cununo macho } \\
\text { bpm140.rx2 }\end{array}$ & $\begin{array}{l}\text { Cununo macho } \\
\text { bpm140.mid }\end{array}$ & $\begin{array}{l}\text { Cununo macho } \\
\text { bpm140.sf2 }\end{array}$ & $\begin{array}{l}\text { Cununo macho } \\
\text { bpm140.wav }\end{array}$ \\
\hline & $\begin{array}{l}\text { Guasá 2_bpm140. } \\
\text { rx2 }\end{array}$ & Guasá 2_bpm140.mid & $\begin{array}{l}\text { Guasá } 2 \\
\text { bpm140.sf2 }\end{array}$ & Guasá 2_bpm140.wav \\
\hline & Guasá_bpm140.rx2 & Guasá_bpm140.mid & $\begin{array}{l}\text { Guasá_bpm140. } \\
\text { sf2 }\end{array}$ & Guasá_bpm140.wav \\
\hline Joropo & $\begin{array}{l}\text { Capachos_bpm230. } \\
\text { rx2 }\end{array}$ & Capachos_bpm230.mid & $\begin{array}{l}\text { Capachos } \\
\text { bpm230.sf2 }\end{array}$ & Capachos_bpm230.wav \\
\hline \multirow{6}{*}{ Pasillo } & Bombo_bpm180.rx2 & Bombo_bpm180.mid & $\begin{array}{l}\text { Bombo_bpm180. } \\
\text { sf2 }\end{array}$ & Bombo_bpm180.wav \\
\hline & $\begin{array}{l}\text { Cucharas_bpm180. } \\
\text { rx2 }\end{array}$ & Cucharas_bpm180.mid & $\begin{array}{l}\text { Cucharas } \\
\text { bpm180.sf2 }\end{array}$ & Cucharas_bpm180.wav \\
\hline & $\begin{array}{l}\text { Guacharaca } \\
\text { bpm180.rx2 }\end{array}$ & $\begin{array}{l}\text { Guacharaca_bpm180. } \\
\text { mid }\end{array}$ & $\begin{array}{l}\text { Guacharaca } \\
\text { bpm180.sf2 }\end{array}$ & $\begin{array}{l}\text { Guacharaca_bpm180. } \\
\text { wav }\end{array}$ \\
\hline & Mate_bpm180.rx2 & Mate_bpm180.mid & $\begin{array}{l}\text { Mate_bpm180. } \\
\text { sf2 }\end{array}$ & Mate_bpm180.wav \\
\hline & Platos_bpm180.rx2 & Platos_bpm180.mid & $\begin{array}{l}\text { Platos_bpm180. } \\
\text { sf2 }\end{array}$ & Platos_bpm180.wav \\
\hline & $\begin{array}{l}\text { Redoblante } \\
\text { bpm180.rx2 }\end{array}$ & $\begin{array}{l}\text { Redoblante_bpm180. } \\
\text { mid }\end{array}$ & $\begin{array}{l}\text { Redoblante- } \\
\text { bpm180.sf2 }\end{array}$ & $\begin{array}{l}\text { Redoblante_bpm180. } \\
\text { wav }\end{array}$ \\
\hline \multirow{3}{*}{ Pelayera } & Bombo_bpm90.rx2 & Bombo_bpm90.mid & $\begin{array}{l}\text { Bombo_bpm90. } \\
\text { sf2 }\end{array}$ & Bombo_bpm90.wav \\
\hline & Platillo_bpm90.rx2 & Platillo_bpm90.mid & $\begin{array}{l}\text { Platillo_bpm90. } \\
\text { sf2 }\end{array}$ & Platillo_bpm90.wav \\
\hline & $\begin{array}{l}\text { Redoblante_bpm90. } \\
\text { rx2 }\end{array}$ & Redoblante_bpm90.mid & $\begin{array}{l}\text { Redoblante } \\
\text { bpm90.st2 }\end{array}$ & Redoblante_bpm90.wav \\
\hline
\end{tabular}




\begin{tabular}{|c|c|c|c|c|}
\hline $\begin{array}{l}\text { Banco de } \\
\text { sonidos }\end{array}$ & RecycleRex file & Midi file & Soundfont & Wave (microsoft) \\
\hline \multirow{6}{*}{$\begin{array}{l}\text { Porro } \\
\text { chocoano }\end{array}$} & Bombo_bpm180.rx2 & Bombo_bpm180.mid & $\begin{array}{l}\text { Bombo_bpm180. } \\
\text { sf2 }\end{array}$ & Bombo_bpm180.wav \\
\hline & $\begin{array}{l}\text { Pate gallina base_ } \\
\text { bpm180.rx2 }\end{array}$ & $\begin{array}{l}\text { Pate gallina base } \\
\text { bpm180.mid }\end{array}$ & $\begin{array}{l}\text { Pate gallina } \\
\text { base_bpm180. } \\
\text { sf2 }\end{array}$ & $\begin{array}{l}\text { Pate gallina base } \\
\text { bpm180.wav }\end{array}$ \\
\hline & $\begin{array}{l}\text { Pate gallina var1_- } \\
\text { bpm180.rx2 }\end{array}$ & $\begin{array}{l}\text { Pate gallina var1__ } \\
\text { bpm180.mid }\end{array}$ & $\begin{array}{l}\text { Pate gallina var1_- } \\
\text { bpm180.sf2 }\end{array}$ & $\begin{array}{l}\text { Pate gallina var1_- } \\
\text { bpm180.wav }\end{array}$ \\
\hline & $\begin{array}{l}\text { Pate gallina var2_ } \\
\text { bpm180.rx2 }\end{array}$ & $\begin{array}{l}\text { Pate gallina var2_ } \\
\text { bpm180.mid }\end{array}$ & $\begin{array}{l}\text { Pate gallina } \\
\text { var2_bpm180.sf2 }\end{array}$ & $\begin{array}{l}\text { Pate gallina var2_ } \\
\text { bpm180.wav }\end{array}$ \\
\hline & $\begin{array}{l}\text { Pate gallina var3_ } \\
\text { bpm180.rx2 }\end{array}$ & $\begin{array}{l}\text { Pate gallina var3_ } \\
\text { bpm180.mid }\end{array}$ & $\begin{array}{l}\text { Pate gallina } \\
\text { var3_bpm180.sf2 }\end{array}$ & $\begin{array}{l}\text { Pate gallina var3_ } \\
\text { bpm180.wav }\end{array}$ \\
\hline & $\begin{array}{l}\text { Platillos_bpm180. } \\
\text { rx2 }\end{array}$ & Platillos_bpm180.mid & $\begin{array}{l}\text { Platillos } \\
\text { bpm180.sf2 }\end{array}$ & Platillos_bpm180.wav \\
\hline \multirow{2}{*}{ Vallenato } & Caja_bpm85.rx2 & Caja_bpm85.mid & Caja_bpm85.sf2 & Caja_bpm85.wav \\
\hline & Guira_bpm85.rx2 & Guira_bpm85.mid & Guira_bpm85.sf2 & Guira_bpm85.wav \\
\hline
\end{tabular}

\section{B. Registro de fonograma}

Este es quizás el producto con mayor versatilidad de uso tradicional; incluso, podría reproducirse desde cualquier plataforma sin importar su procedencia o especificaciones. En este formato se sintetizan todas las capturas separadas por tracks de reproducción dependiendo del aire capturado. Los primeros presentan los bucles o loops

de cada aire y región incluyendo el BPM de la toma y al final termina con un track dedicado a las tomas o golpes sencillos de cada instrumento [9].

En la Tabla $V$ se puede observar el listado con los temas de reproducción.

TABLA V

Listado de Temas de Reproducción del Fonograma [7]

\begin{tabular}{|c|l|l|l|}
\hline $\begin{array}{c}\text { Track } \\
\text { List }\end{array}$ & \multicolumn{1}{|c|}{ Nombre } & \multicolumn{1}{c|}{ Contenido } & \multicolumn{1}{c|}{ Tiempo } \\
\hline & \multicolumn{1}{|c|}{ Región andina } & 00:04 \\
\hline 1 & Región andina bambuco_160 & Loop completo, 4 compases en 3/4, & $00: 33$ \\
\hline 2 & $\begin{array}{l}\text { Región andina bambuco-instru- } \\
\text { mentos_bpm160 }\end{array}$ & Loop instrumentos, & $00: 03$ \\
\hline 3 & Región andina pasillo_180 & Loop completo, 4 compases en 3/4, & $00: 25$ \\
\hline 4 & $\begin{array}{l}\text { Región andina pasillo-instrumen- } \\
\text { tos_bpm180 }\end{array}$ & Loop instrumentos, & $00: 54$ \\
\hline 5 & Región andina-samples & Loop instrumentos, & \multicolumn{1}{|c|}{. } \\
\hline
\end{tabular}




\begin{tabular}{|c|c|c|c|}
\hline $\begin{array}{c}\text { Track } \\
\text { List }\end{array}$ & Nombre & Contenido & Tiempo \\
\hline \multicolumn{4}{|c|}{ Región atlántica } \\
\hline 6 & Región atlántica cumbia_bpm185 & $\begin{array}{l}\text { Loop completo con anacrusa, } 4 \text { compases } \\
\text { en } 2 / 2\end{array}$ & 00:05 \\
\hline 7 & $\begin{array}{l}\text { Región atlántica cumbia con ale- } \\
\text { gre_bpm185 }\end{array}$ & $\begin{array}{l}\text { Loop completo con anacrusa, } 8 \text { compases } \\
\text { en } 2 / 2\end{array}$ & 00:10 \\
\hline 8 & $\begin{array}{l}\text { Región atlántica cumbia-instru- } \\
\text { mentos_bpm185 }\end{array}$ & Loop instrumentos, & $00: 53$ \\
\hline 9 & Región atlántica pelayera_bpm180 & Loop completo, 4 compases en $4 / 4$ & 00:10 \\
\hline 10 & $\begin{array}{l}\text { Región atlántica pelayera-instru- } \\
\text { mentos_bpm180 }\end{array}$ & Loop instrumentos, & 00:32 \\
\hline 11 & $\begin{array}{l}\text { Región atlántica vallenato-aire de } \\
\text { son_bpm85 }\end{array}$ & $\begin{array}{l}\text { Loop completo, } 8 \text { compases, 4/4. Aire de } \\
\text { son }\end{array}$ & 00:11 \\
\hline 12 & $\begin{array}{l}\text { Región atlántica vallenato-aire de } \\
\text { son-instrumentos_bpm85 }\end{array}$ & $\begin{array}{l}\text { Loop instrumentos, } 8 \text { compases, } 4 / 4 \text {. Aire } \\
\text { de son }\end{array}$ & $00: 23$ \\
\hline 13 & Región atlántica cumbia-samples & Loop instrumentos, & $00: 57$ \\
\hline 14 & Región atlántica pelayera-samples & Loop instrumentos, & $00: 32$ \\
\hline 15 & Región atlántica vallenato-samples & Loop instrumentos, & 00:09 \\
\hline \multicolumn{4}{|c|}{ Región pacífica } \\
\hline 16 & $\begin{array}{l}\text { Región pacífica porro chocoa- } \\
\text { no_bpm90 }\end{array}$ & Loop completo, 4 compases, $2 / 2$ & 00:05 \\
\hline 17 & $\begin{array}{l}\text { Región pacífica porro chocoano- } \\
\text { variación 1_bpm90 }\end{array}$ & Loop completo, 4 compases, $2 / 2$ & 00:05 \\
\hline 18 & $\begin{array}{l}\text { Región pacífica porro chocoano- } \\
\text { variación 2_bpm90 }\end{array}$ & Loop completo, 4 compases, $2 / 2$ & 00:05 \\
\hline 19 & $\begin{array}{l}\text { Región pacífica porro chocoano- } \\
\text { variación 3_bpm90 }\end{array}$ & Loop completo, 4 compases, $2 / 2$ & 00:05 \\
\hline 20 & $\begin{array}{l}\text { Región pacífica porro chocoano- } \\
\text { instrumentos_bpm90 }\end{array}$ & Loop instrumentos, & $00: 34$ \\
\hline 21 & Región pacífica currulao_bpm140 & $\begin{array}{l}\text { Loop completo con anacrusa, } 4 \text { compases, } \\
6 / 8\end{array}$ & 00:05 \\
\hline 22 & $\begin{array}{l}\text { Región pacífica currulao-instru- } \\
\text { mentos_bpm140 }\end{array}$ & Loop instrumentos, & $00: 32$ \\
\hline 23 & $\begin{array}{l}\text { Región pacífica porro chocoano- } \\
\text { samples }\end{array}$ & Loop instrumentos, & $00: 32$ \\
\hline 24 & Región pacífica currulao-samples & Loop instrumentos, & $00: 42$ \\
\hline
\end{tabular}




\begin{tabular}{|c|l|l|l|}
\hline $\begin{array}{c}\text { Track } \\
\text { List }\end{array}$ & \multicolumn{1}{|c|}{ Nombre } & \multicolumn{1}{|c|}{ Contenido } & \multicolumn{1}{|c|}{ Tiempo } \\
\hline 25 & $\begin{array}{l}\text { Región llanos orientales joro- } \\
\text { po_bpm230 }\end{array}$ & $\begin{array}{l}\text { Loop completo con anacrusa, 4 compases, } \\
6 / 8\end{array}$ & $00: 06$ \\
\hline 26 & $\begin{array}{l}\text { Región insular calypso_bpm110 } \\
\text { Loop completo, 4 compases, 2/2 }\end{array}$ & $00: 04$ \\
\hline 27 & $\begin{array}{l}\text { Región insular calypso-instrumen- } \\
\text { tos_bpm110 }\end{array}$ & Loop instrumentos, & $00: 18$ \\
\hline 28 & $\begin{array}{l}\text { Región insular llanos orientales } \\
\text { joropo-instrumentos }\end{array}$ & Loop instrumentos, & $00: 03$ \\
\hline
\end{tabular}

Aplicación móvil: esta aplicación, diseñada con el objetivo de tener un instrumento de divulgación y conocimiento de los ritmos colombianos para el público en general, fue programada con base en el sistema Unity 3D, de tal forma, que tuviese una versatilidad cercana al ambiente de videojuego, para lograr, así, una mayor apropiación social del conocimiento específico derivado de este proyecto de investigación. En las Figs. 1, 2, 3 y 4 se muestra el mapa general de la aplicación en su primera versión básica 1.0.

Desde la pantalla inicial se puede acceder en forma general a la totalidad de ritmos, los cuales están divididos de acuerdo con la región específica.

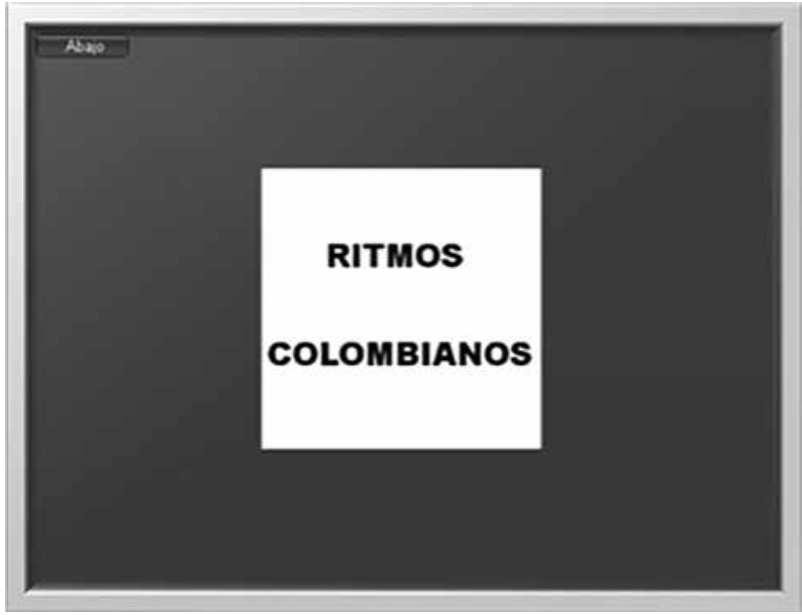

Fig. 1 Pantalla principal de la aplicación.

En la pantalla secundaria se observan desplegadas las regiones geográficas del país en donde se encuentra cada aire musical de cada región.

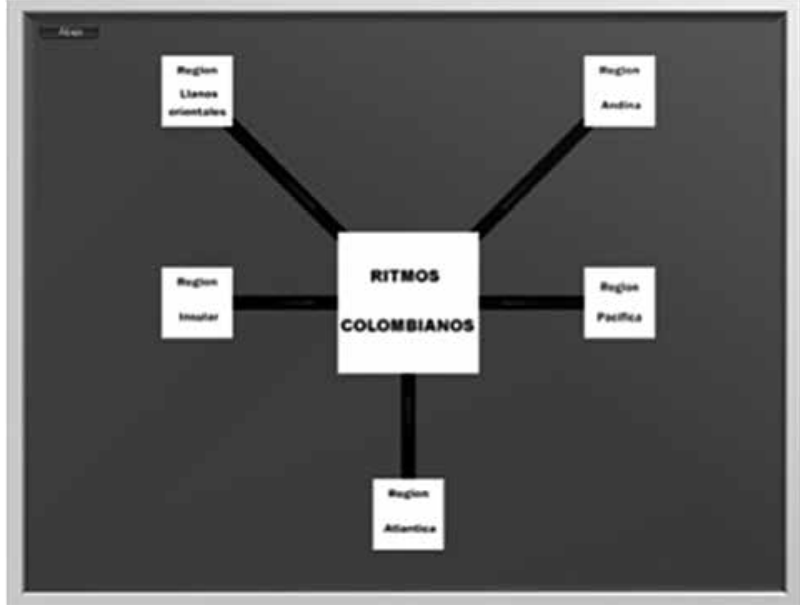

Fig. 2 Pantalla secundaria de la aplicación.

En esta sección se despliegan los ritmos característicos de cada región.

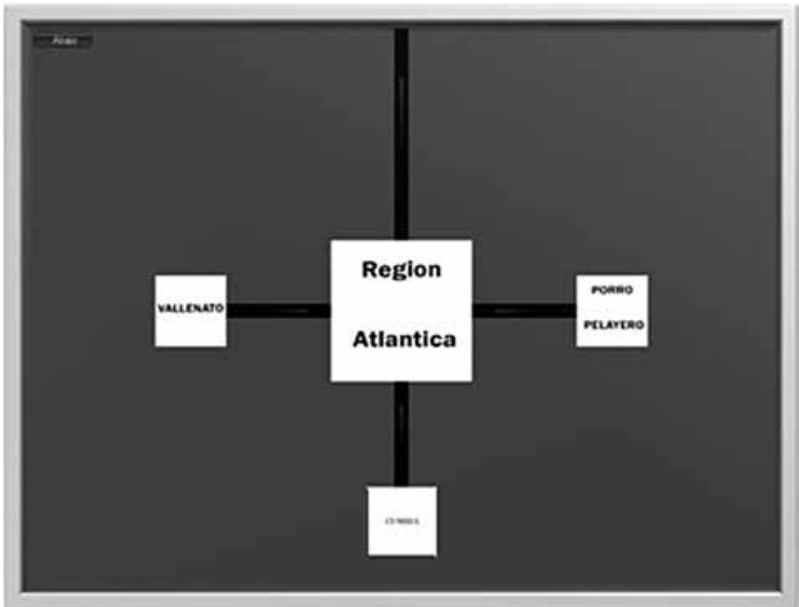

Fig. 3 Pantalla de cada región geográfica.

En la última ventana se pueden apreciar los instrumentos característicos de cada región. 
En el ejemplo se observa la ventana del ritmo de vallenato la cual, a su vez, despliega los dos instrumentos típicos de percusión que utiliza. Además, tiene la posibilidad de reproducir el ritmo en modo de bucle o loop desde la ventana del ritmo y cada instrumento de manera independiente con la misma base rítmica [10].

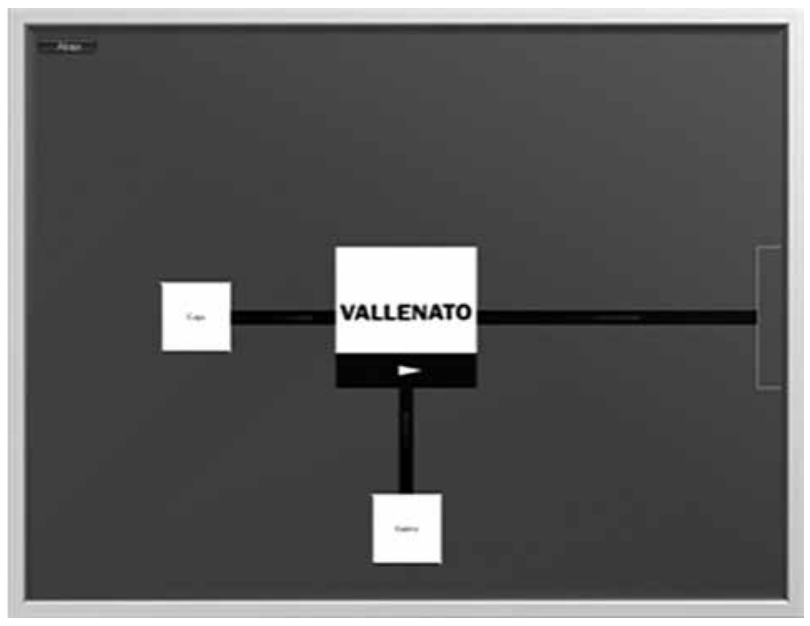

Fig. 4 Despliegue de la ventana del ritmo específico.

\section{Conclusiones}

Si bien, los resultados evidencian tres tipos de productos de divulgación, también muestran una clara metodología de trabajo tanto para la captura como para la interpretación musical necesaria que conduzca a obtener un registro básico requerido para la creación de cualquier banco virtual de instrumentos de percusión. Vale aclarar, que aunque esta nos sirve especialmente para los instrumentos del folclor colombiano, puede ser fácilmente adaptada a cualquier otro aire o género musical.

Otra de las importantes conclusiones tiene que ver con el resultado en sí de las muestras, las cuales fueron realizadas en un estudio de ambiente y acústica controlado y dieron como resultado un sonido bastante fiel al esperado. Cabe anotar la importancia que tiene el intérprete de los instrumentos, quien, para este caso, fue un músico experto en cada uno de los aires. La importancia del intérprete radica no solamente en la ejecución profesional de los instrumentos o instrumento, sino también en la disposición para la realización de cada muestra, debido a que la grabación difiere bastante de una convencional, en la cual se tiene un mapa predeterminado de lo que se quiere efectuar. En este caso se debe interpretar analizando las diferentes posibilidades interpretativas que sugiere la metodología de trabajo propuesta en la investigación.

En cuanto al desarrollo del software, es importante recalcar la necesidad de seguir explorando nuevas posibilidades y versiones de la aplicación realizada, pues esta se llevó a cabo como trabajo de grado de un estudiante investigador perteneciente a un pregrado en tecnología y se hace necesario la evolución de la misma, no solo en diseño, sino también en su alcance funcional.

La oportunidad que nos brinda la creación de esta base de datos, obliga necesariamente a la configuración de un repositorio digital con todas y cada una de las tomas, de tal forma que esté al alcance de investigadores, músicos, productores y cualquier otra persona interesada en la exploración de los instrumentos percutidos del folclor colombiano. Así mismo, surge la necesidad de sumarse a una red digital en el ámbito latinoamericano, que facilite compartir y descargar los bancos de sonidos, para, que de esta manera, cualquier músico o investigador en otro país pueda hacer música con nuestros instrumentos y al generarse el intercambio, se logre tener al alcance sonidos de otras culturas y, por ende, experimentar con estos.

Por último, vale la pena recordar parte de la pregunta que se debe responder, en el caso de la falta de bancos virtuales de instrumentos musicales pertenecientes al folclor colombiano. A pesar de que en gran parte se resuelve, es necesario profundizar en otros tipos de instrumentos melódicos y armónicos de nuestro folclor, así como también evolucionar cada uno de los instrumentos percutidos ya registrados en esta misma investigación. 


\section{Referencias}

[1] BARTLETT, Bruce; BARTLETT, Jenny. Practical recording techniques. the step-by approach to professsional audio recording. 5th ed. Burlington, Mass: Focal Press/Elsevier, 2009. Pag. 101-163; 404-406.

[2] GIBSON, Bill. Instrument \& vocal recording. 1st Ed. New York: Hal Leonard, 2007. Pag. 89-129.

[3] OWSINSKI, Bobby. The mixing engineer's handbook. 2nd ed. Boston: Course technology, Cengage Learning, 2006. Pag. 24-37.

[4] MIYARA, Federico. Acústica y sistemas de sonidos. 4a ed. Buenos Aires : Universidad Nacional de Rosario, 2006.

[5] JARAMILLO JARAMILLO, Ana María. Acústica. la ciencia del sonido. 1a ed. Medellín: Instituto Tecnológico Metropolitano, 2007.

[6] GIBSON, Bill. Recording software \& plug-ins. 1st ed. New York: Hal Leonard, 2007. Pag. 46-66; 101-121; 183-194.
[7] GIBSON, Bill. Mixing \& mastering. 1st ed. New York : Hal Leonard, 2007. Pag. 61-72.

[8] CABALLERO PARRA, Carlos Andrés. La producción musical en estudio. 1a ed. Medellín: Instituto Tecnológico Metropolitano, 2010.

[9] HUBER, David Miles; RUNSTEIN, Robert E. Modern recording techniques. 7th ed. Burlington, Mass: Focal Press/Elsevier, 2010. Pag. 133-179; 291-391.

[10] IZHAKI, Roey. Mixing audio. Concepts, practices and tools. 7th ed. Burlington, Mass: Focal Press/Elsevier, 2010. Pag. 29-72.

[11] OWSINSKI, Bobby. The recording engineer's handbook. 2nd ed. Boston: Course technology, Cengage Learning, 2009. Pag. 87-95. 
